\title{
Long working hours are associated with unmet dental needs in south Korean male adults who have experienced dental pain
}

\author{
Yitak Kim ${ }^{1 \dagger}$, Sangwon Lee ${ }^{1 \dagger}$, Juyeong $\mathrm{Kim}^{2 \dagger}$, Eun-Cheol Park ${ }^{3,4}$ and Sung-In Jang ${ }^{3,4^{*}}$
}

\begin{abstract}
Backgrounds: We explored the association between working hours and unmet dental needs among adults who have experienced dental pain, and how this relationship varied by demographic and lifestyle factors.

Methods: We used the data of 9594 adults who reported dental pain from the Korea National Health and Nutrition Examination Survey (KNHANES) $V$ and VI. We conducted a logistic regression analysis to determine the association between working hours and unmet dental needs, followed by a subgroup analysis and Cochran-Armitage trend tests.

Results: Among the 4203 male subjects, 1661 (39.5\%) experienced unmet dental needs. They also showed a significant dose-response relationship between working hours and unmet dental needs (OR 1.21 [95\% Cl 0.97-1.51], OR 1.30 [95\% Cl 0.99-1.69], OR 1.33 [95\% Cl 1.04-1.71], OR 1.58 [95\% Cl 1.21-2.07] compared to no working hours), whereas female participants did not. The significance of the association was preserved among participants with increased consumption of alcohol, urban residence, and who brushed their teeth at least twice a day. It was also stronger among those who lacked access to dental services or did not perceive the need for dental care.

Conclusion: Among adults who have experienced dental pain, unmet dental needs had higher odds of occurring in males who worked longer, and this relationship appears to be influenced by consumption of alcohol, region of residence, tooth-brushing frequency, and access to and perception of dental care. Accordingly, policies should be drafted to reduce unmet needs by considering these factors.
\end{abstract}

Keywords: Unmet needs, Dental pain, Unmet dental needs, Working hours, Dose-response relationship, Region, Alcohol

\section{Background}

A good society ensures that individuals can readily obtain appropriate medical services when needed. Accordingly, many countries strive to invest in medical facilities [1]. However, improving the medical facilities does not always translate to a better hospital experience for patients. In fact, quantitative expansion in medicine-such as constructing new hospitals and improving medical

\footnotetext{
* Correspondence: JANGSI@yuhs.ac

†Yitak Kim, Sangwon Lee and Juyeong Kim contributed equally to this work.

${ }^{3}$ Institute of Health Services Research, Yonsei University, Seoul, Republic of Korea

${ }^{4}$ Department of Preventive Medicine, Yonsei University College of Medicine, 50-1 Yonsei-ro, Seodaemun-gu, Seoul 120-752, Republic of Korea Full list of author information is available at the end of the article
}

facilities-appears to have little effect on patients if patients cannot effectively reach a doctor [2].

A variety of obstacles can hinder individuals from reaching or deciding to contact a doctor, even when they might need to [3]. Unmet needs in health care can lead to a range of adverse health outcomes [4]. By identifying and resolving the causes of unmet needs in patients, we can expect an improvement in overall medical services without further investment in medical resources, which are often limited. Canada has noted a number of diverse efforts in considering gender, income, and social integration to alleviate inequalities in unmet dental needs [5].

In South Korea, the ratio of dental expenses to total medical expenses is rising rapidly, which accords with the dangerously high rate of unmet dental needs among

(c) The Author(s). 2019 Open Access This article is distributed under the terms of the Creative Commons Attribution 4.0 International License (http://creativecommons.org/licenses/by/4.0/), which permits unrestricted use, distribution, and 
both children and adults (20 and 40\%, respectively) [6], Alarmingly, the rate of unmet dental needs is almost twice that of other diseases [6]. Thus, it is very important to determine and eliminate the factors that contribute to this high rate of unmet dental needs.

The excessive working hours among Korean adults is a contentious topic in Korean society. Among the Organization for Economic Co-operation and Development (OECD) countries, Korea was ranked third in terms of annual working hours in 2016 [7]. Many studies have pointed out that long working hours can have a range of adverse effects, including a higher incidence of physical problems such as diabetes mellitus and metabolic syndrome, as well as psychological problems such as anxiety and excessive alcohol use $[8,9]$. It is similarly possible that longer working hours plays a role in unmet care needs, with overworking leading to less time available for accessing medical services. If this is found, reducing overall working hours might help improve the overall healthcare system.

There are several previous studies on the factors associated with unmet dental needs, conducted in both Western countries and South Korea [10, 11]. However, few of these studies utilized dental pain as an index of unmet dental needs, and most were limited to Western countries and child populations [10]. One study in the United States of America (USA) examined adults to find out factors of unmet dental needs, but they did not look for associations [12]. Also, studies examining the association between working hours and unmet dental needs among people who have experienced dental pain were generally unfound. This suggests the need for such study within this specific Korean population. By utilizing an objective index of illness (i.e., dental pain), we might be able to resolve the subjectivity of the unmet needs variable [13], which is often mentioned as a limitation in former studies $[14,15]$. These objective indicators could certify the actual demands of patients, and more precisely detect health inequity. Therefore, we chose a sample exclusively comprised of subjects who have experienced dental pain, given that toothache is an unbiased index of the need for dental care [16].

We hypothesized that longer working hours will be associated with greater unmet needs among workers who have experienced dental pain. Furthermore, we performed a subgroup analysis with demographic and behavioral variables to examine which factors influence this association. Factors such as region of residence can affect the relationship, due to different accessibility to medical facilities.

\section{Methods}

\section{Study design and participants}

We used the data from the Korea National Health and Nutrition Examination Survey (KNHANES) V and VI, a nationwide cross-sectional study conducted from 2010 to 2015 by the Korean Ministry of Health and Welfare. The research population is homogeneous and unbiased, and represents non-institutionalized Korean civilians [17]. For this study, we selected adults older than 19 years old with valid responses to all items and had experienced dental pain. Among the 39,518 participants, after excluding participants with missing values and adolescents, we chose 10,118 respondents who reported the experience of dental pain. After further eliminating participants with invalid answers to regular dental checkups $(n=104)$, national health insurance $(n=85)$, drinking habits $(n=27)$, occupation $(n=305)$, and education $(n=$ $3), 9594$ respondents were analyzed.

\section{Working hours}

Respondents were asked about their weekly working hours with the question "How long do you work per week, including extra work/night shift and excluding mealtimes?" The Labor Standards Act of Korea states that standard working hours must not exceed $40 \mathrm{~h}$ per week; work up to $48-60 \mathrm{~h}$ is defined as "extra work" and is given extra wages [18]. Studies have reported that people who work longer than $60 \mathrm{~h}$ per week tend to suffer from health problems such as higher cardiovascular mortality rates [19]. Accordingly, we classified participants' answers to this question into four groups: $40 \mathrm{~h}$ or less, 41-48, 49-60, and 61 or more.

\section{Unmet needs}

Unmet dental needs were assessed with two questions. First, they were asked "Have you ever wanted to visit a dentist but could not?". Those who answered 'yes' were then asked why, and the answers were classified into three groups. Firstly, 'Lack of ability to pay' was classified as 'economic reasons'. Secondly, 'Dental clinic is too far away,' 'could not leave workplace or school,' 'mobility or health problems,' and 'had to take care of children' were classified as 'lack of access'. Finally, 'did not consider it a serious problem' and 'afraid to visit a dentist' were classified as 'perceptual barriers' [20].

\section{Other variables}

Demographic variables included gender, age (20-29, 30 $39,40-49,50-59,60-65$, and $>65$ years), and region of residence (urban or rural areas). The socioeconomic variables included level of education (middle school or lower, high school, and college or higher), occupation type (office, labor, and service), and household income (high, moderately high, moderately low, and low). Household income was divided into quartiles using the monthly average equivalent household income (i.e., monthly household income divided by the square root of the number of household members) [17]. The health- 
related variables considered were smoking (non-smoker, past smoker, and present smoker), drinking habits (nondrinker, light drinker, and heavy drinker), possession of private insurance, health insurance type (national health insurance (NHI)-local, NHI-employee's, and medical aid), self-rated oral health status (good, moderate, bad). Heavy drinkers were defined as those who drank $>2$ times a week, light drinkers as those who drank less than twice per week, and non-drinkers as those who never drank or drank less than once per month. Finally, the dental care indicators included usage of dental care tools, number of times of teeth brushing per day, and dental checkup within the last year. Unless otherwise mentioned, the above variables were binary variables (yes or no).

\section{Statistical analysis}

Because average workload, occupation, and physical abilities differ considerably according to gender [21], we stratified all analysis by gender. For binary variables, we calculated the frequency and proportions of each variable and compared them using chi-square tests. The association was quantified using logistic regression analyses after adjusting for demographic, socioeconomic, health-related, and dental care indicators. Additionally, we performed subgroup analyses according to drinking habits, region of residence, and tooth-brushing habit. Cochran-Armitage trend tests were used to determine the $\mathrm{p}$ for trend between working hours and unmet dental needs. For this test, working hours were defined as a continuous variable (with an interval of $1 \mathrm{~h}$ ) and unmet dental needs as a binary variable. All analyses were conducted using SAS 9.4 (SAS Inc., Cary, NC, USA). There were no human subjects involved in this study.

\section{Results}

Table 1 displays the general characteristics of the gender-stratified study population. Of the 4203 (43.8\%) male participants and $5391(56.2 \%)$ female participants, 1661 (39.5\%) and 2376 (44.1\%) had experienced unmet dental needs, respectively. Among both males and females, the percentage of unmet dental needs increased with working hours $(p<0.001$ in males, $p=0.017$ in female). Specifically, among males, the proportions of unmet dental needs were $32.3,38.5,40.9,41.7$, and $46.9 \%$ in the $0,<40,41-48,49-60$, and $>60$ h groups, respectively; among females, the proportions of unmet dental needs were $41.3,45.2,46.2,46.3$, and $48.2 \%$, respectively.

Table 2 presents the logistic regression analysis results adjusted with confounding factors for both males and females. We observed a dose-response relationship between working hours and unmet need only in male participants. Specifically, the odds ratios (ORs) and 95\% confidence intervals (CI) for the working hour groups (vs. the 0-h group) were as follows: OR $=1.21[95 \% \mathrm{CI}$ $0.97-1.51$ ] for $<40 \mathrm{~h}$; OR $=1.30$ [95\% CI 0.99-1.69] for $41-48 \mathrm{~h}$; OR $=1.33$ [95\% CI 1.04-1.71] for $49-60 \mathrm{~h}$; and $\mathrm{OR}=1.58$ [95\% CI 1.21-2.07] for $>60 \mathrm{~h}$. In other words, the odds ratios increased with working hours among males. Among females, the ORs showed a bell-shaped pattern $(\mathrm{OR}=1.24$ [95\% CI 1.08-1.42] for $<40 \mathrm{~h}$; OR = 1.27 [95\% CI 1.02-1.58] for $41-48 \mathrm{~h}$; OR $=1.24$ [95\% CI $1.00-1.54$ ] for $49-60 \mathrm{~h}$; OR $=1.21$ [95\% CI $0.95-1.55$ ] for $>60 \mathrm{~h}$ ).

The subgroup analysis is shown in Table 3 , separately for males and females. For alcohol consumption, males defined as heavy drinkers showed significantly higher ORs (for the 41-48, 49-60, and $>60 \mathrm{~h}$ ) and maintained the dose-response relationship. Among females, the ORs increased with working hours in the light drinking group particularly, although significance was found only for the $1-40,41-48$, and $49-60 \mathrm{~h}$ groups. As for region of residence, males continued to show a dose-response relationship in urban areas $(\mathrm{OR}=1.17$ [95\% CI 0.90-1.51] for < $40 \mathrm{~h}$; OR $=1.39$ [95\% CI 1.02-1.90] for $41-48 \mathrm{~h}$; $\mathrm{OR}=1.52 \quad[95 \% \mathrm{CI} 1.14-2.03]$ for $49-60 \mathrm{~h} ; \mathrm{OR}=1.65$ [95\% CI 1.21-2.25] for $>60 \mathrm{~h}$ ), but not in rural areas. As for times tooth-brushing habit, both males and females showed a stronger positive relationship between working hours and unmet dental need when they brushed at least twice a day.

Table 4 shows the associations between working hours and unmet dental needs for each reason group. For both males and females, participants who lacked access to dental care and had perceptual barriers for dental care showed higher ORs than did those who lacked the ability to pay. Furthermore, among participants who lacked access, the relationship between working hours and unmet dental needs remained positive; in contrast, the relationship was negative among those who had perceptual barriers for dental care.

\section{Discussion}

The purpose of this study was to examine the association between working hours and unmet dental needs in a specific population of Koreans-those with experience of dental pain. We also conducted subgroup analyses by alcohol consumption level, region of residence, toothbrushing frequency, and the major reasons for unmet dental needs.

We observed a dose-response relationship between working hours and unmet dental needs in the male group. In order to interpret effect sizes of odds ratios, the odds ratios were converted into Cohen's d, or the standardized mean difference between two group means [22]. While the effect size of the odds ratio of the $>60 \mathrm{~h}$ group $(\mathrm{OR}=1.54$, or Cohen's $\mathrm{d}=0.2)$ is considered small, it is nevertheless reflective of a continued stepwise 
Table 1 General characteristics by unmet dental need

\begin{tabular}{|c|c|c|c|c|c|c|c|c|c|c|c|c|}
\hline \multirow[t]{3}{*}{ Variable } & \multirow[t]{3}{*}{$\mathrm{N}$} & \multirow[t]{3}{*}{$\%$} & \multicolumn{5}{|c|}{ Male $(n=4173)$} & \multicolumn{5}{|c|}{ Female $(n=5355)$} \\
\hline & & & \multicolumn{2}{|c|}{ No unmet need } & \multicolumn{2}{|c|}{ Unmet need } & \multirow[b]{2}{*}{$p$-value } & \multicolumn{2}{|c|}{ No unmet need } & \multicolumn{2}{|c|}{ Unmet need } & \multirow[b]{2}{*}{$p$-value } \\
\hline & & & $\mathrm{N}$ & $\%$ & $\mathrm{~N}$ & $\%$ & & $\mathrm{~N}$ & $\%$ & $\mathrm{~N}$ & $\%$ & \\
\hline Total & 9528 & 100.0 & 2524 & 60.5 & 1649 & 39.5 & & 2995 & 55.9 & 2360 & 44.1 & \\
\hline Working hours per week & & & & & & & $<0.001$ & & & & & 0.030 \\
\hline No work & 2816 & 29.6 & 510 & 67.5 & 245 & 32.5 & & 1208 & 58.6 & 853 & 41.4 & \\
\hline$<40$ & 3346 & 35.1 & 844 & 61.6 & 527 & 38.4 & & 1083 & 54.8 & 892 & 45.2 & \\
\hline $41-48$ & 1100 & 11.5 & 365 & 59.1 & 253 & 40.9 & & 259 & 53.7 & 223 & 46.3 & \\
\hline $49-60$ & 1366 & 14.3 & 516 & 58.2 & 371 & 41.8 & & 258 & 53.9 & 221 & 46.1 & \\
\hline$>60$ & 900 & 9.4 & 289 & 53.3 & 253 & 46.7 & & 187 & 52.2 & 171 & 47.8 & \\
\hline Age & & & & & & & $<0.001$ & & & & & 0.160 \\
\hline $20-29$ & 1076 & 11.3 & 245 & 59.3 & 168 & 40.7 & & 353 & 53.2 & 310 & 46.8 & \\
\hline $30-39$ & 1678 & 17.6 & 370 & 54.2 & 313 & 45.8 & & 537 & 54.0 & 458 & 46.0 & \\
\hline $40-49$ & 1692 & 17.8 & 448 & 59.7 & 303 & 40.3 & & 539 & 57.3 & 402 & 42.7 & \\
\hline $50-59$ & 2019 & 21.2 & 527 & 56.4 & 407 & 43.6 & & 595 & 54.8 & 490 & 45.2 & \\
\hline $60-65$ & 1058 & 11.1 & 311 & 64.8 & 169 & 35.2 & & 335 & 58.0 & 243 & 42.0 & \\
\hline $65-$ & 2005 & 21.0 & 623 & 68.3 & 289 & 31.7 & & 636 & 58.2 & 457 & 41.8 & \\
\hline Private insurance & & & & & & & 0.404 & & & & & 0.184 \\
\hline No & 2551 & 26.8 & 711 & 61.5 & 445 & 38.5 & & 759 & 54.4 & 636 & 45.6 & \\
\hline Yes & 6977 & 73.2 & 1813 & 60.1 & 1204 & 39.9 & & 2236 & 56.5 & 1724 & 43.5 & \\
\hline Health insurance type & & & & & & & $<0.001$ & & & & & $<0.001$ \\
\hline $\mathrm{NHI}$ (local) & 3215 & 33.7 & 841 & 57.4 & 623 & 42.6 & & 921 & 52.6 & 830 & 47.4 & \\
\hline NHI (employee's) & 5971 & 62.7 & 1629 & 62.7 & 969 & 37.3 & & 1966 & 58.3 & 1407 & 41.7 & \\
\hline Medical aid & 342 & 3.6 & 54 & 48.6 & 57 & 51.4 & & 108 & 46.8 & 123 & 53.2 & \\
\hline Household income & & & & & & & 0.035 & & & & & $<0.001$ \\
\hline Low & 1817 & 19.1 & 418 & 58.0 & 303 & 42.0 & & 564 & 51.5 & 532 & 48.5 & \\
\hline low - moderate & 2471 & 25.9 & 634 & 59.9 & 425 & 40.1 & & 764 & 54.1 & 648 & 45.9 & \\
\hline Moderate -High & 2599 & 27.3 & 693 & 59.1 & 479 & 40.9 & & 816 & 57.2 & 611 & 42.8 & \\
\hline High & 2641 & 27.7 & 779 & 63.8 & 442 & 36.2 & & 851 & 59.9 & 569 & 40.1 & \\
\hline Region of residence & & & & & & & 0.001 & & & & & 0.736 \\
\hline Urban & 7417 & 77.8 & 2005 & 61.8 & 1239 & 38.2 & & 2339 & 56.1 & 1834 & 43.9 & \\
\hline Rural & 2111 & 22.2 & 519 & 55.9 & 410 & 44.1 & & 656 & 55.5 & 526 & 44.5 & \\
\hline Occupation type & & & & & & & 0.065 & & & & & 0.308 \\
\hline Office & 3417 & 35.9 & 919 & 62.8 & 544 & 37.2 & & 1108 & 56.7 & 846 & 43.3 & \\
\hline Labor & 5181 & 54.4 & 1464 & 59.1 & 1015 & 40.9 & & 1514 & 56.0 & 1188 & 44.0 & \\
\hline Service & 930 & 9.8 & 141 & 61.0 & 90 & 39.0 & & 373 & 53.4 & 326 & 46.6 & \\
\hline Self-assessment of dental health & & & & & & & $<0.001$ & & & & & $<0.001$ \\
\hline Good & 716 & 7.5 & 297 & 83.4 & 59 & 16.6 & & 286 & 79.4 & 74 & 20.6 & \\
\hline Moderate & 3071 & 32.2 & 890 & 71.9 & 347 & 28.1 & & 1226 & 66.8 & 608 & 33.2 & \\
\hline Bad & 5741 & 60.3 & 1337 & 51.8 & 1243 & 48.2 & & 1483 & 46.9 & 1678 & 53.1 & \\
\hline Reason for unmet dental needs* & & & & & & & $<0.001$ & & & & & $<0.001$ \\
\hline No unmet need & 5519 & 57.9 & 2524 & 100.0 & 0 & 0.0 & & 2995 & 100.0 & 0 & 0.0 & \\
\hline Lack of ability to pay & & & 0 & 0.0 & 590 & 35.8 & & 0 & 0.0 & 931 & 39.4 & \\
\hline Lack of ability to reach & & & 0 & 0.0 & 543 & 32.9 & & 0 & 0.0 & 617 & 43.2 & \\
\hline Lack of ability to perceive & & & 0 & 0.0 & 516 & 31.3 & & 0 & 0.0 & 812 & 28.1 & \\
\hline
\end{tabular}


Table 1 General characteristics by unmet dental need (Continued)

\begin{tabular}{|c|c|c|c|c|c|c|c|c|c|c|c|c|}
\hline \multirow[t]{3}{*}{ Variable } & \multirow[t]{3}{*}{$\mathrm{N}$} & \multirow[t]{3}{*}{$\%$} & \multicolumn{5}{|c|}{ Male $(n=4173)$} & \multicolumn{5}{|c|}{ Female $(n=5355)$} \\
\hline & & & \multicolumn{2}{|c|}{ No unmet need } & \multicolumn{2}{|c|}{ Unmet need } & \multirow[b]{2}{*}{$p$-value } & \multicolumn{2}{|c|}{ No unmet need } & \multicolumn{2}{|c|}{ Unmet need } & \multirow[b]{2}{*}{$p$-value } \\
\hline & & & $\mathrm{N}$ & $\%$ & $\mathrm{~N}$ & $\%$ & & $\mathrm{~N}$ & $\%$ & $\mathrm{~N}$ & $\%$ & \\
\hline Number of family members & & & & & & & 0.018 & & & & & 0.063 \\
\hline More than one & 8654 & 90.8 & 2367 & 61.0 & 1515 & 39.0 & & 2690 & 56.4 & 2082 & 43.6 & \\
\hline Alone & 874 & 9.2 & 157 & 54.0 & 134 & 46.0 & & 305 & 52.3 & 278 & 47.7 & \\
\hline Level of education & & & & & & & 0.020 & & & & & 0.644 \\
\hline Middle school & 3457 & 36.3 & 758 & 60.8 & 488 & 39.2 & & 1223 & 55.3 & 988 & 44.7 & \\
\hline High school & 3049 & 32.0 & 829 & 57.8 & 606 & 42.2 & & 902 & 55.9 & 712 & 44.1 & \\
\hline$\geq$ college & 3022 & 31.7 & 937 & 62.8 & 555 & 37.2 & & 870 & 56.9 & 660 & 43.1 & \\
\hline Usage of dental care tools & & & & & & & $<0.001$ & & & & & $<0.001$ \\
\hline No & 5363 & 56.3 & 1481 & 56.9 & 1120 & 43.1 & & 1423 & 51.5 & 1339 & 48.5 & \\
\hline Yes & 4165 & 43.7 & 1043 & 66.3 & 529 & 33.7 & & 1572 & 60.6 & 1021 & 39.4 & \\
\hline Number of times brushing teeth per day & & & 0.004 & & & & & 0.069 & & & & \\
\hline $0-1$ & 1240 & 13.0 & 419 & 55.9 & 331 & 44.1 & & 255 & 52.0 & 235 & 48.0 & \\
\hline$\geq 2$ & 8288 & 87.0 & 2105 & 61.5 & 1318 & 38.5 & & 2740 & 56.3 & 2125 & 43.7 & \\
\hline Dental checkup within last one year & & & & & & & $<0.001$ & & & & & $<0.001$ \\
\hline No & 6546 & 68.7 & 1596 & 56.9 & 1207 & 43.1 & & 1900 & 50.8 & 1843 & 49.2 & \\
\hline Yes & 2982 & 31.3 & 928 & 67.7 & 442 & 32.3 & & 1095 & 67.9 & 517 & 32.1 & \\
\hline Smoke & & & & & & & $<0.001$ & & & & & $<0.001$ \\
\hline No & 5424 & 56.9 & 500 & 67.1 & 245 & 32.9 & & 2666 & 57.0 & 2013 & 43.0 & \\
\hline Current smoker & 2104 & 22.1 & 947 & 53.7 & 815 & 46.3 & & 162 & 47.4 & 180 & 52.6 & \\
\hline Past smoker & 2000 & 21.0 & 1077 & 64.6 & 589 & 35.4 & & 167 & 50.0 & 167 & 50.0 & \\
\hline Drink & & & & & & & 0.148 & & & & & 0.938 \\
\hline No drink & 3579 & 37.6 & 444 & 61.5 & 278 & 38.5 & & 1110 & 56.2 & 864 & 43.8 & \\
\hline Light drink & 2363 & 24.8 & 1135 & 61.7 & 704 & 38.3 & & 1594 & 55.8 & 1263 & 44.2 & \\
\hline Heavy drink & 2136 & 22.4 & 945 & 58.6 & 667 & 41.4 & & 291 & 55.5 & 233 & 44.5 & \\
\hline Average hours of sleep per week & & & & & & & 0.036 & & & & & $<0.001$ \\
\hline$<5$ & 478 & 5.0 & 86 & 55.1 & 70 & 44.9 & & 159 & 49.4 & 163 & 50.6 & \\
\hline $5-6$ & 3772 & 39.6 & 980 & 58.4 & 698 & 41.6 & & 1125 & 53.7 & 969 & 46.3 & \\
\hline $7-8$ & 4588 & 48.2 & 1297 & 62.5 & 777 & 37.5 & & 1474 & 58.6 & 1040 & 41.4 & \\
\hline$>8$ & 690 & 7.2 & 161 & 60.8 & 104 & 39.2 & & 237 & 55.8 & 188 & 44.2 & \\
\hline BMI & & & & & & & 0.113 & & & & & 0.130 \\
\hline$\leq 25$ & 4275 & 44.9 & 1552 & 59.6 & 1054 & 40.4 & & 2087 & 56.6 & 1599 & 43.4 & \\
\hline$>25$ & 3236 & 34.0 & 972 & 62.0 & 595 & 38.0 & & 908 & 54.4 & 761 & 45.6 & \\
\hline Year & & & & & & & $<0.001$ & & & & & $<0.001$ \\
\hline 2010 & 1489 & 15.6 & 357 & 54.0 & 304 & 46.0 & & 419 & 50.6 & 409 & 49.4 & \\
\hline 2011 & 1335 & 14.0 & 329 & 57.1 & 247 & 42.9 & & 408 & 53.8 & 351 & 46.2 & \\
\hline 2012 & 1789 & 18.8 & 459 & 58.2 & 329 & 41.8 & & 530 & 52.9 & 471 & 47.1 & \\
\hline 2013 & 1769 & 18.6 & 486 & 62.6 & 290 & 37.4 & & 589 & 59.3 & 404 & 40.7 & \\
\hline 2014 & 1529 & 16.0 & 448 & 67.4 & 217 & 32.6 & & 497 & 57.5 & 367 & 42.5 & \\
\hline 2015 & 1617 & 17.0 & 445 & 62.9 & 262 & 37.1 & & 552 & 60.7 & 358 & 39.3 & \\
\hline
\end{tabular}

* The ratios of each reason represents the percentage compared to the number of people who showed unmet needs 
Table 2 Adjusted odds ratios for factors associated with unmet dental need

\begin{tabular}{llll}
\hline Variable & $\frac{\text { Male }(p<0.001)^{*}}{\text { Unmet need }}$ & & Female $(p=0.001)^{*}$ \\
$\quad 95 \% \mathrm{Cl}$ & & OR $\quad 95 \% \mathrm{Cl}$
\end{tabular}

Working hours per week

\begin{tabular}{lllllll} 
no work & 1.00 & \multicolumn{5}{c}{1.00} \\
$<40$ & 1.21 & 0.97 & 1.51 & 1.23 & 1.07 & 1.41 \\
$41-48$ & 1.29 & 0.98 & 1.68 & 1.26 & 1.01 & 1.56 \\
$49-60$ & 1.32 & 1.03 & 1.71 & 1.22 & 0.98 & 1.52 \\
$>60$ & 1.54 & 1.17 & 2.02 & 1.16 & 0.90 & 1.48
\end{tabular}

Age

$\begin{array}{lllllll}20-29 & 1.00 & & & 1.00 & & \\ 30-39 & 1.24 & 0.94 & 1.64 & 1.04 & 0.84 & 1.29 \\ 40-49 & 0.92 & 0.70 & 1.21 & 0.90 & 0.72 & 1.12 \\ 50-59 & 1.00 & 0.76 & 1.31 & 0.99 & 0.78 & 1.26 \\ 60-65 & 0.73 & 0.53 & 1.00 & 0.78 & 0.58 & 1.04 \\ 65- & 0.57 & 0.42 & 0.78 & 0.63 & 0.47 & 0.85\end{array}$

Private insurance

\begin{tabular}{lllllll} 
No & 1.00 & \multicolumn{5}{c}{1.00} \\
Yes & 0.97 & 0.81 & 1.17 & 1.00 & 0.85 & 1.17
\end{tabular}

Health insurance type

\begin{tabular}{lllllll} 
NHI (local) & 1.00 & \multicolumn{1}{c}{1.00} \\
NHI (employee's) & 0.90 & 0.78 & 1.04 & 0.86 & 0.76 & 0.97 \\
Medical aid & 1.33 & 0.86 & 2.06 & 1.16 & 0.85 & 1.57
\end{tabular}

Household income

\begin{tabular}{lllllll} 
Low & 1.00 & \multicolumn{5}{c}{1.00} \\
low - moderate & 0.85 & 0.68 & 1.07 & 0.89 & 0.74 & 1.08 \\
Moderate -High & 0.89 & 0.70 & 1.12 & 0.81 & 0.66 & 0.99 \\
High & 0.76 & 0.59 & 0.97 & 0.79 & 0.64 & 0.97
\end{tabular}

Region of residence

$\begin{array}{lcccccc}\text { Urban } & 1.00 & & & 1.00 & & \\ \text { Rural } & 1.26 & 1.07 & 1.48 & 0.95 & 0.82 & 1.09 \\ \text { Occupation type } & & & & & & \\ \text { Office } & 1.00 & & & 1.00 & & \\ \text { Labor } & 0.93 & 0.78 & 1.10 & 0.84 & 0.70 & 1.00 \\ \text { Service } & 0.84 & 0.61 & 1.16 & 0.95 & 0.77 & 1.17 \\ \text { Self-assessment of dental health } & & & & & \\ \text { Good } & 1.00 & & & 1.00 & & \\ \text { Moderate } & 1.80 & 1.31 & 2.46 & 1.94 & 1.47 & 2.56 \\ \text { Bad } & 4.22 & 3.14 & 5.68 & 4.41 & 3.36 & 5.78\end{array}$

Number of family members

$\begin{array}{lllllll}\text { More than one } & 1.00 & & & 1.00 & & \\ \text { Alone } & 1.21 & 0.93 & 1.58 & 1.11 & 0.91 & 1.36 \\ \text { Level of education } & & & & & & \\ \text { Middle school } & 1.00 & & & 1.00 & & \end{array}$

Table 2 Adjusted odds ratios for factors associated with unmet dental need (Continued)

\begin{tabular}{|c|c|c|c|c|c|c|}
\hline \multirow[t]{3}{*}{ Variable } & \multicolumn{3}{|c|}{ Male $(p<0.001)^{*}$} & \multicolumn{3}{|c|}{ Female $(p=0.001)^{*}$} \\
\hline & \multicolumn{3}{|c|}{ Unmet need } & \multicolumn{3}{|c|}{ Unmet need } \\
\hline & \multirow{2}{*}{$\begin{array}{l}\mathrm{OR} \\
1.18\end{array}$} & \multicolumn{2}{|c|}{$95 \% \mathrm{Cl}$} & \multirow{2}{*}{$\begin{array}{l}\mathrm{OR} \\
1.08\end{array}$} & \multicolumn{2}{|c|}{$95 \% \mathrm{Cl}$} \\
\hline High school & & 0.97 & 1.43 & & 0.89 & 1.31 \\
\hline$\geq$ college & 1.05 & 0.84 & 1.32 & 1.16 & 0.91 & 1.48 \\
\hline \multicolumn{7}{|c|}{ Usage of dental care tools } \\
\hline No & 1.00 & & & 1.00 & & \\
\hline Yes & 0.74 & 0.64 & 0.86 & 0.75 & 0.66 & 0.85 \\
\hline
\end{tabular}

Number of times brushing teeth per day

\begin{tabular}{lllllll}
$0-1$ & 1.00 & \multicolumn{5}{c}{1.00} \\
$\geq 2$ & 0.85 & 0.72 & 1.02 & 0.89 & 0.73 & 1.10
\end{tabular}

Dental checkup within last one year

$\begin{array}{lllllll}\text { No } & 1.00 & & & 1.00 & & \\ \text { Yes } & 0.68 & 0.59 & 0.79 & 0.51 & 0.44 & 0.58\end{array}$

Smoke

No $\quad 1.00$

$\begin{array}{lllllll}\text { Current smoker } & 1.39 & 1.15 & 1.70 & 1.20 & 0.94 & 1.53\end{array}$

$\begin{array}{lllllll}\text { Past smoker } & 1.19 & 0.97 & 1.45 & 1.28 & 1.01 & 1.62\end{array}$

Drink

\begin{tabular}{lllllll} 
No drink & 1.00 & \multicolumn{5}{c}{1.00} \\
Light drink & 0.87 & 0.71 & 1.05 & 0.96 & 0.84 & 1.09 \\
Heavy drink & 0.89 & 0.73 & 1.09 & 0.87 & 0.70 & 1.07
\end{tabular}

Average hours of sleep per week

$\begin{array}{ccccccc}<5 & 1.00 & & & 1.00 & & \\ 5-6 & 0.80 & 0.56 & 1.14 & 0.88 & 0.68 & 1.13 \\ 7-8 & 0.68 & 0.48 & 0.97 & 0.72 & 0.56 & 0.93 \\ >8 & 0.67 & 0.44 & 1.03 & 0.72 & 0.52 & 0.98 \\ \text { BMI } & & & & & & \\ \leq 25 & 1.00 & & & 1.00 & & \\ >25 & 0.89 & 0.78 & 1.02 & 1.03 & 0.91 & 1.17 \\ \text { Year } & & & & & & \\ 2010 & 1.21 & 0.96 & 1.53 & 1.28 & 1.05 & 1.57 \\ 2011 & 1.11 & 0.87 & 1.41 & 1.17 & 0.95 & 1.44 \\ 2012 & 1.10 & 0.88 & 1.38 & 1.29 & 1.06 & 1.56 \\ 2013 & 0.94 & 0.75 & 1.18 & 1.06 & 0.87 & 1.28 \\ 2014 & 0.81 & 0.64 & 1.02 & 1.15 & 0.94 & 1.40 \\ 2015 & 1.00 & & & 1.00 & & \end{array}$

* These $p$-values represent the result of the Cochran-Armitage trend test for each subgroup

association among males. No association between working hours and unmet dental needs was observed for females. The potential cause of this relationship in males is the early closing time of hospitals in South Korea. In other words, when a salaried worker visits the hospital after work (usually at $5 \mathrm{pm}$ ), there is little time for them 
Table 3 Association between work hours and unmet need by different factors

\begin{tabular}{|c|c|c|c|c|c|c|c|}
\hline \multirow[t]{3}{*}{ Variable } & & \multirow{2}{*}{\multicolumn{3}{|c|}{$\frac{\text { Male }}{\text { Unmet need }}$}} & \multirow{2}{*}{\multicolumn{3}{|c|}{$\frac{\text { Female }}{\text { Unmet need }}$}} \\
\hline & & & & & & & \\
\hline & & \multirow[t]{2}{*}{$\overline{\mathrm{OR}}$} & \multicolumn{2}{|l|}{$95 \% \mathrm{Cl}$} & OR & \multicolumn{2}{|l|}{$95 \% \mathrm{Cl}$} \\
\hline \multicolumn{7}{|l|}{ Drink } & \\
\hline \multirow[t]{5}{*}{ No drink } & $\mathrm{Oh}$ & 1.00 & $p=0.011^{*}$ & & & \multicolumn{2}{|l|}{$p=0.066$} \\
\hline & $1-40 \mathrm{~h}$ & 0.99 & 0.62 & 1.58 & 1.19 & 0.95 & 1.48 \\
\hline & 40-48 h & 1.32 & 0.70 & 2.50 & 1.06 & 0.72 & 1.58 \\
\hline & $48-60 \mathrm{~h}$ & 1.10 & 0.61 & 1.98 & 1.00 & 0.66 & 1.51 \\
\hline & $\geq 61 \mathrm{~h}$ & 1.71 & 0.91 & 3.24 & 1.42 & 0.93 & 2.19 \\
\hline \multirow[t]{5}{*}{ Light drink } & $\mathrm{Oh}$ & 1.00 & \multicolumn{2}{|l|}{$p=0.002$} & & \multicolumn{2}{|l|}{$p=0.007$} \\
\hline & $1-40 \mathrm{~h}$ & 1.15 & 0.82 & 1.62 & 1.27 & 1.05 & 1.54 \\
\hline & $40-48 \mathrm{~h}$ & 1.12 & 0.75 & 1.68 & 1.38 & 1.03 & 1.85 \\
\hline & $48-60 \mathrm{~h}$ & 1.23 & 0.84 & 1.80 & 1.44 & 1.07 & 1.93 \\
\hline & $\geq 61 \mathrm{~h}$ & 1.56 & 1.02 & 2.39 & 1.11 & 0.78 & 1.56 \\
\hline \multirow[t]{5}{*}{ Heavy drink } & $\mathrm{Oh}$ & 1.00 & \multicolumn{2}{|l|}{$p<0.001$} & & \multicolumn{2}{|l|}{$p=0.204$} \\
\hline & $1-40 \mathrm{~h}$ & 1.44 & 0.97 & 2.15 & 1.19 & 0.74 & 1.91 \\
\hline & $40-48 \mathrm{~h}$ & 1.61 & 1.01 & 2.57 & 1.43 & 0.73 & 2.81 \\
\hline & $48-60 \mathrm{~h}$ & 1.69 & 1.08 & 2.65 & 1.07 & 0.55 & 2.08 \\
\hline & $\geq 61 \mathrm{~h}$ & 1.63 & 1.03 & 2.59 & 0.84 & 0.40 & 1.76 \\
\hline
\end{tabular}

Region of residence

\begin{tabular}{llllllll} 
Urban & $0 h$ & 1.00 & $\mathbf{p}<\mathbf{0 . 0 0 1}$ & \multicolumn{5}{c}{$\mathbf{p = 0 . 0 0 2}$} \\
& $1-40 h$ & 1.16 & 0.90 & 1.51 & 1.20 & 1.02 & 1.40 \\
& $40-48 h$ & 1.37 & 1.01 & 1.87 & 1.22 & 0.95 & 1.55 \\
& $48-60 h$ & 1.53 & 1.14 & 2.04 & 1.31 & 1.01 & 1.70 \\
& $\geq 61 h$ & 1.60 & 1.17 & 2.18 & 1.12 & 0.83 & 1.51 \\
\multirow{4}{*}{ Rural } & $0 h$ & 1.00 & $\boldsymbol{p}=\mathbf{0 . 1 1 4}$ & & & $\boldsymbol{p}=\mathbf{0 . 2 1 5}$ \\
& $1-40 \mathrm{~h}$ & 1.35 & 0.86 & 2.13 & 1.47 & 1.08 & 1.98 \\
& $40-48 \mathrm{~h}$ & 1.00 & 0.57 & 1.74 & 1.54 & 0.95 & 2.50 \\
& $48-60 \mathrm{~h}$ & 0.83 & 0.49 & 1.42 & 1.28 & 0.83 & 1.96 \\
& $\geq 61 \mathrm{~h}$ & 1.39 & 0.79 & 2.45 & 1.44 & 0.90 & 2.29
\end{tabular}

Number of times brushing teeth per day

\begin{tabular}{|c|c|c|c|c|c|c|c|}
\hline \multirow[t]{2}{*}{$0-1$} & $\mathrm{Oh}$ & 1.00 & $p=0.019$ & & & \multicolumn{2}{|l|}{$p=0.362$} \\
\hline & $1-40 \mathrm{~h}$ & 1.41 & 0.89 & 2.23 & 1.01 & 0.64 & 1.59 \\
\hline & $40-48$ h & 1.17 & 0.62 & 2.20 & 0.85 & 0.33 & 2.18 \\
\hline & $48-60 h$ & 1.20 & 0.67 & 2.16 & 0.78 & 0.36 & 68 \\
\hline & $\geq 61 \mathrm{~h}$ & 1.67 & 0.93 & 3.00 & 1.37 & 0.65 & 2.89 \\
\hline \multirow[t]{5}{*}{$\geq 2$} & $\mathrm{Oh}$ & 1.00 & $p<0.001$ & & & \multicolumn{2}{|l|}{$p=0.001$} \\
\hline & $1-40 \mathrm{~h}$ & 1.18 & 0.91 & 1.52 & 1.26 & 1.09 & \\
\hline & $40-48$ h & 1.30 & 0.96 & 1.76 & 1.30 & 1.04 & 53 \\
\hline & $48-60 h$ & 1.37 & 1.03 & 1.83 & 1.30 & 1.03 & \\
\hline & $\geq 61 \mathrm{~h}$ & 1.56 & 1.14 & 2.13 & 1.15 & 0.88 & .50 \\
\hline
\end{tabular}

* Bolded numbers represent the p-value of the Cochran-Armitage trend test for each subgroup to see a doctor before the hospital closes (typically around $6 \mathrm{pm}$ ). Given that South Korea has long working hours (ranked 3rd place among OECD countries) [7], it is feasible that longer working hours leads to greater unmet needs.

Unlike a previous study [15], females did not show a positive relationship between working hours and unmet care needs. However, participants in this study all had experienced dental pain, unlike of participants in Soek et al. The time periods when the data was collected are also different. The differing results suggest the validity of gender stratification in studies on employment status $[21,23]$. Gender difference in workplaces can also lead to different job roles and positions, even when the occupation type and working hours are the same [21]. Greater unmet needs can imply either of the following: higher unmet with identical needs, or superior demands in the first place. The former might be explained by conventional gender roles, whereby men tend to gain more stress from loss of job opportunities and job-related failures [24]. Accordingly, men might experience greater pressure at their workplaces, which hinders them from taking sick leave. The latter can be understood by considering that males have higher health-related concerns, including metabolic syndromes and suicide rates [23, 25]. Regardless of the explanations, stricter regulation policies for working hours appear to be more necessary for males.

The subgroup analysis for alcohol consumption in Table 3 indicates that there are generally significant associations between working hours and unmet dental needs among heavy drinkers for males and light drinkers for females. Drinking habits are linked to decreased risk awareness, and alcohol-related disorders require social treatments [26, 27]. Thus, excessive alcohol consumption can mislead people from receiving proper medication, which relates to the significance of the results. The higher ORs in the light drinking group among females is perhaps due to the lower tolerance of alcohol in women compared to men [28].

Region of residence has been highlighted as a controversial factor in recent years [29-31]. Rural areas have markedly different characteristics from urban areas, including a more restricted labor market, closed social network, and limited health-related resources [30]. Previous studies have shown both higher and comparable levels of unmet needs in rural regions (vs. urban ones) among Americans [30, 31]. Conversely, a study in the Korean population claimed that urban residents experience greater unmet care needs for outpatient care, after adjusting for other factors [29]. These discrepancies potentially result from the differing cultures and healthcare systems. Our findings are consistent with Kim et al.'s [29], with the urban subgroup showing a significant 
Table 4 Association by different reasons of unmet need

\begin{tabular}{|c|c|c|c|c|c|c|c|}
\hline \multirow[t]{3}{*}{ Variable } & & \multicolumn{3}{|c|}{ Male } & \multicolumn{3}{|c|}{ Female } \\
\hline & & \multicolumn{3}{|c|}{ Unmet need } & \multicolumn{3}{|c|}{ Unmet need } \\
\hline & & \multirow[t]{2}{*}{$\mathrm{OR}$} & \multicolumn{2}{|l|}{$95 \% \mathrm{Cl}$} & \multirow[t]{2}{*}{ OR } & \multicolumn{2}{|l|}{$95 \% \mathrm{Cl}$} \\
\hline \multicolumn{6}{|l|}{ Reasons of unmet need } & & \\
\hline \multirow[t]{5}{*}{ Lack of ability to pay } & $\mathrm{Oh}$ & 1.00 & $<0.001^{*}$ & & 1.00 & $<0.001$ & \\
\hline & $1-40 h$ & 1.08 & 0.74 & 1.58 & 1.08 & 0.86 & 1.35 \\
\hline & $40-48 \mathrm{~h}$ & 1.26 & 0.80 & 1.98 & 1.08 & 0.76 & 1.54 \\
\hline & $48-60 h$ & 0.89 & 0.57 & 1.37 & 0.69 & 0.48 & 0.99 \\
\hline & $\geq 61 \mathrm{~h}$ & 1.26 & 0.81 & 1.97 & 0.84 & 0.58 & 1.23 \\
\hline \multirow[t]{5}{*}{ Lack of ability to reach } & $\mathrm{Oh}$ & 1.00 & $<0.001$ & & 1.00 & $<0.001$ & \\
\hline & $1-40 h$ & 1.43 & 0.91 & 2.24 & 1.14 & 0.89 & 1.46 \\
\hline & $40-48 \mathrm{~h}$ & 1.68 & 1.02 & 2.76 & 2.12 & 1.50 & 2.98 \\
\hline & $48-60 \mathrm{~h}$ & 2.80 & 1.75 & 4.48 & 3.21 & 2.28 & 4.51 \\
\hline & $\geq 61 \mathrm{~h}$ & 2.36 & 1.45 & 3.84 & 2.30 & 1.56 & 3.40 \\
\hline \multirow[t]{5}{*}{ Lack of ability to perceive } & $\mathrm{Oh}$ & 1.00 & 0.004 & & 1.00 & $<0.001$ & \\
\hline & $1-40 \mathrm{~h}$ & 0.70 & 0.48 & 1.03 & 0.81 & 0.65 & 1.01 \\
\hline & $40-48$ h & 0.53 & 0.34 & 0.83 & 0.45 & 0.32 & 0.64 \\
\hline & $48-60 \mathrm{~h}$ & 0.40 & 0.26 & 0.62 & 0.43 & 0.30 & 0.61 \\
\hline & $\geq 61 \mathrm{~h}$ & 0.35 & 0.22 & 0.56 & 0.54 & 0.36 & 0.80 \\
\hline
\end{tabular}

* Bolded numbers represent the p-value of the Cochran-Armitage trend test for each subgroup

dose-response relationship. Despite the proximity of hospitals in urban regions, urban residents' lack of time might lead to greater unmet needs [15].

Interestingly, participants with a higher frequency of tooth brushing showed greater ORs and stronger relationship between working hours and unmet dental needs. This contradicts the commonsense notion that regular tooth brushing improves oral health [32]. However, in this study, more brushing might imply greater effort to improve health status, since all participants had experienced dental pain. These individuals might have higher expectations in their health, thus elevating their demand and strengthening the relationship between working hours and dental unmet need. Past studies support this idea, where privately insured people showed greater unmet needs [33].

In this study, lack of access and perceptual barriers for dental care were significant moderators of the relationship between working hours and unmet dental need. These results are consistent with previous papers demonstrating that a lack of time is a major reason for unmet needs $[15,33]$. The negative relationship between working hours and unmet needs in those who had perceptual barriers for dental care can be explained by the subjectivity of unmet needs, which are highly personal [34]. Specifically, when an unmet need is not perceived as a need by the person-for instance, when individuals perceive working is more important than visiting the doctor-the actual demand will decrease, thus leading to the negative association between working hours and unmet needs. The fact that unmet needs are not solely the result of economic burden supports the idea that society itself must do more to improve overall healthcare quality than mere financial investment.

Our results have several policy implications. First, the association between working hours and unmet dental needs, and the fact that lack of access (including time) is a major reason for unmet, indicate the importance of time in health care. To ensure adequate health care access, policies should be put in place to reduce timerelated barriers, such as extending sick-leave breaks or changing hospital hours. Region should also be considered in these policies. Previous studies in Canada and South Ethiopia evaluating differences along the ruralurban continuum have sought to utilize primary health care systems to reduce unmet needs [35, 36]. These former findings suggest feasible strategies for urban areas with long working hours. Lastly, this study is also likely to support the field of preventive dentistry by precluding unmet dental needs in advance.

This study has several limitations. First, cross-sectional data cannot infer any causal relationships. Therefore, caution is required in interpreting our results. Second, this study excluded relevant occupation-related variables: the sample was stratified according to working hours (including non-working group) but did not account for nightshift work or wage type due to collinearity. Further research should include these variables as confounders. 
Third, we did not include all possible oral health-related factors, such as history of dental diseases, oral health education and medication, because these specific variables were not included in KNHANES questionnaire. Lastly, our manipulation of the occupation variable might disturb the validity of the results; while it preserved $30 \%$ of the population, further studies are needed to prove its validity.

Nevertheless, this study endeavored to remain nationally representative by utilizing 6 years of longitudinal data and a large sample. In addition, the sample contained non-workers and all had experience of dental pain, meaning that the population was refined. Furthermore, plausible mechanisms for obscure issues were demonstrated by taking advantage of up-to-date evidence.

\section{Conclusion}

Only male subjects showed significant dose-response relationships between working hours and unmet dental needs. In addition, increased consumption of alcohol, residing in urban areas, brushing teeth at least twice a day, and lacking access and perception of the need for dental care significantly moderated the relationships between working hours and unmet dental needs. Future research controlling for other occupation variables would further solidify our results and offer more practical implications for workplaces in South Korea.

\section{Abbreviations}

Cl: Confidence Interval; h, hr.: hour; KNHANES: Korea National Health and Nutrition Examination Survey; NHI: National Health Insurance;

OECD: Organization for Economic Co-Operation and Development; OR: Odds Ratio; USA: United States of America

\section{Acknowledgements}

Not applicable.

\section{Authors' contributions}

YTK, SWL, and JYK contributed to the research design, data analysis, and interpretation of data. YTK, SWL, SIJ and JYK carried out the interpretation of data. ECP reviewed the article. YTK and SWL wrote the article. All authors have read and approved the manuscript.

\section{Funding}

This research received no specific grant from any funding agency in public commercial or not-for-profit sectors.

\section{Availability of data and materials}

KNHANES $V$ and $V I$ data can be accessed from the KNHANES homepage (URL: https://knhanes.cdc.go.kr/knhanes/eng/index.do).

\section{Ethics approval and consent to participate}

KNHANES is openly published. Thus, ethical approval was not required for this study. This study did not require informed consent from the participants, as their information was fully anonymized and unidentified prior to analysis. KNHANES data was approved by the Institutional Review Board of the Korean Centers for Disease Control and Prevention (IRB No. 2010-02CON-21C, 2011-02CON-06-C, 2012-01EXP-01-2C, 2013-07CON-03-4C, 2013-12EXP-035C).
Consent for publication

Not applicable.

\section{Competing interests}

The authors report no conflicts of interest in this work.

\section{Author details}

${ }^{1}$ College of Medicine, Yonsei University, Seoul, Republic of Korea. ${ }^{2}$ Department of Health \& Human Performance, Sahmyook University, Seoul, Republic of Korea. ${ }^{3}$ Institute of Health Services Research, Yonsei University, Seoul, Republic of Korea. ${ }^{4}$ Department of Preventive Medicine, Yonsei University College of Medicine, 50-1 Yonsei-ro, Seodaemun-gu, Seoul 120-752, Republic of Korea.

Received: 6 September 2019 Accepted: 8 November 2019

Published online: 21 November 2019

\section{References}

1. Kapferer S. The importance of investing in health. In. World Economic Forum: World Economic Forum; 2015

2. Staff Writer. TOP 10: countries investing the Most on health care. Healthcare Global: In; 2014

3. Sanmartin C, Houle C, Tremblay S, Berthelot JM. Changes in unmet health care needs. Health Rep. 2002;13(3):15-21.

4. Ko H. Unmet healthcare needs and health status: panel evidence from Korea. Health Policy. 2016;120(6):646-53.

5. Bryant T, Leaver C, Dunn J. Unmet healthcare need, gender, and health inequalities in Canada. Health Policy. 2009;91 (1):24-32.

6. Jung H: Korean National Health Accounts in 2014. In. Edited by Ministry of Health and Welfare: Ministry of Health and Welfare, 2015.

7. Hours worked. https://data.oecd.org/emp/hours-worked.htm.

8. Kivimaki M, Virtanen M, Kawachi I, Nyberg ST, Alfredsson L, Batty GD, Bjorner JB, Borritz M, Brunner EJ, Burr H, et al. Long working hours, socioeconomic status, and the risk of incident type 2 diabetes: a meta-analysis of published and unpublished data from 222120 individuals. The lancet Diabetes \& endocrinology. 2015;3(1):27-34

9. Bannai A, Tamakoshi A. The association between long working hours and health: a systematic review of epidemiological evidence. Scand J Work Environ Health. 2014;40(1):5-18

10. Villalobos-Rodelo JJ, Medina-Solis CE, Maupome G, Lamadrid-Figueroa H, Casanova-Rosado AJ, Casanova-Rosado JF, Marquez-Corona Mde L. Dental needs and socioeconomic status associated with utilization of dental services in the presence of dental pain: a case-control study in children. J Orofac Pain. 2010;24(3):279-86.

11. Chae S. Factors associated with perceived unmet dental care needs of older adults unmet dental care needs. Geriatr Gerontol Int. 2017;17(11):1936-42.

12. Caban-Martinez AJ, Lee DJ, Fleming LE, Arheart KL, LeBlanc WG, ChungBridges K, Christ S, Pitman T: Dental care access and unmet dental care needs among U.S. workers: the National Health Interview Survey, 1997 to 2003. The Journal of the American Dental Association 2007, 138(2):227-230.

13. Bebbington PE, Meltzer $H$, Brugha TS, Farrell M, Jenkins R, Ceresa C, Lewis $G$. Unequal access and unmet need: neurotic disorders and the use of primary care services. Psychol Med. 2000:30(6):1359-67.

14. Popovic N, Terzic-Supic Z, Simic S, Mladenovic B. Predictors of unmet health care needs in Serbia; analysis based on EU-SILC data. PLoS One. 2017;12(11): e0187866.

15. Soek H. A dose-response relationship between long working hours and unmet need for access to hospital facilities. Scand J Work Environ Health. 2016;42(2):135-43.

16. Lewis C, Stout J. Toothache in us children. Archives of Pediatrics \& Adolescent Medicine. 2010;164(11):1059-63.

17. Kweon S, Kim Y. Jang M-j, Kim Y, Kim K, Choi S, Chun C, Khang Y-H, oh K: data resource profile: the Korea National Health and nutrition examination survey (KNHANES). Int J Epidemiol. 2014;43(1):69-77.

18. Ministry of Government Legislation: Labor Standards Act. In. Edited by Ministry of Employment and Labor. Seoul; 2010: Article 50.

19. Spurgeon A, Harrington JM, Cooper CL. Health and safety problems associated with long working hours: a review of the current position. Occup Environ Med. 1997;54(6):367-75. 
20. Levesque JF, Harris MF, Russell G. Patient-centred access to health care: conceptualising access at the interface of health systems and populations. Int J Equity Health. 2013;12:18.

21. Park JW, Park JS, Kim S, Park M, Choi H, Lim S. The association between long working hours and hearing impairment in noise unexposed workers: data from the 5th Korea National Health and nutrition examination survey (KNHANES 2010-2012). Annals of occupational and environmental medicine. 2016;28:55.

22. Chen $\mathrm{H}$, Cohen $\mathrm{P}$, Chen $\mathrm{S}$. How big is a big odds ratio? Interpreting the magnitudes of odds ratios in epidemiological studies. Communications in Statistics - Simulation and Computation. 2010;39(4):860-4.

23. Myong J-P, Kim H-R, Jung-Choi K, Baker D, Choi B. Disparities of metabolic syndrome prevalence by age, gender and occupation among Korean adult workers. Ind Health. 2012;50(2):115-22.

24. Cutright $P$. The relative gender gap in suicide: societal integration, the culture of suicide, and period effects in 20 developed countries, 1955-1994. Soc Sci Res. 2001;30(1):76-99.

25. Kim SY. Comparative epidemiology of suicide in South Korea and Japan: effects of age, gender and suicide methods. Crisis : the journal of crisis intervention and suicide prevention. 2011;32(1):5-14.

26. Steptoe A, Wardle J. Health behaviour, risk awareness and emotional wellbeing in students from Eastern Europe and Western Europe. Soc Sci Med. 2001;53(12):1621-30

27. Mojtabai R, Crum RM. Perceived unmet need for alcohol and drug use treatments and future use of services: results from a longitudinal study. Drug Alcohol Depend. 2013;127(1-3):59-64

28. Nolen-Hoeksema S. Gender differences in risk factors and consequences for alcohol use and problems. Clin Psychol Rev. 2004;24(8):981-1010.

29. Kim J, Kim TH, Park EC, Cho WH: Factors influencing unmet need for health care services in Korea. Asia-Pacific journal of public health 2015, 27(2): Np2555-2569.

30. Peterson: County-Level Poverty Is Equally Associated With Unmet Health Care Needs in Rural and Urban Settings. The Journal of rural health 2010 26(4):373.

31. Merwin E, Snyder A, Katz E. Differential access to quality rural healthcare: professional and policy challenges. Family \& Community Health. 2006;29(3): 186-94

32. Buglar ME, White KM, Robinson NG. The role of self-efficacy in dental patients' brushing and flossing: testing an extended health belief model. Patient Educ Couns. 2010;78(2):269-72.

33. Lee S-Y. Unmet healthcare needs depending on employment status. Health policy (Amsterdam). 2015;119(7):899-906.

34. Allin S: Subjective unmet need and utilization of health care services in Canada: What are the equity implications? Social science \& medicine (1982) 2010, 70(3):465-472.

35. Sibley LM, Weiner JP. An evaluation of access to health care services along the rural-urban continuum in Canada. BMC Health Serv Res. 2011;11:20.

36. Mekonnen W, Worku A: Determinants of low family planning use and high unmet need in Butajira District, South Central Ethiopia Reproductive Health 2011, 8(1):37.

\section{Publisher's Note}

Springer Nature remains neutral with regard to jurisdictional claims in published maps and institutional affiliations.

\section{Ready to submit your research? Choose BMC and benefit from:}

- fast, convenient online submission

- thorough peer review by experienced researchers in your field

- rapid publication on acceptance

- support for research data, including large and complex data types

- gold Open Access which fosters wider collaboration and increased citations

- maximum visibility for your research: over $100 \mathrm{M}$ website views per year

At $\mathrm{BMC}$, research is always in progress.

Learn more biomedcentral.com/submissions 